\title{
O DIÁLOGO ENTRE O PÓS-MÉTODO E A COMPLEXIDADE: CRIANDO OPORTUNIDADES DE APRENDIZAGEM NA SALA DE AULA
}

\section{Establishing a dialogue between the post-method condition and complexity: creating learning opportunities in the classroom}

\author{
Maria Eugenia Witzler D’ESPOSITO \\ Faculdade Cultura Inglesa, São Paulo, São Paulo, Brasil \\ Maria Antonieta Alba CELANI \\ Pontifícia Universidade Católica de São Paulo, São Paulo, São Paulo, Brasil
}

\begin{abstract}
RESUMO: Neste artigo teórico resenhamos e discutimos a respeito da mudança paradigmática na educação (MORIN, 1990/2008; BEHRENS \& OLIARI, 2007; MORAES, 2006) e, a partir dela, repensamos o ensino-aprendizagem e a prática docente. Para tal, estabelecemos um diálogo entre a condição pós-método (Kumaravadivelu, 2006) e a perspectiva da complexidade (Morin, 1990/2008, 1999/2006a, 1999/2006b, 2005/2006, 2008/2010), examinando a inter-relação entre os parâmetros pedagógicos da condição pós-método, as oportunidades de aprendizagem (CRABBE, 2003 e ALLWRIGHT, 2005) e a metodologia de projetos (BEHRENS, 2006).
\end{abstract}

PALAVRAS-CHAVE: Oportunidades de aprendizagem; Condição pós-método; Complexidade; Parâmetros pedagógicos da condição pós-método; Metodologia de projetos

\begin{abstract}
In this theoretical paper we present and discuss the change in the education paradigm (MORIN, 1990/2008; BEHRENS \& OLIARI, 2007; MORAES, 2006) and, having it as a starting point, we rethink the teaching-learning and the teaching practice. In order to do so, we establish a dialogue between the post-method condition (Kumaravadivelu, 2006) and the complexity perspective (Morin, 1990/2008, 1999/2006a, 1999/2006b, 2005/2006, 2008/2010), examining the interrelationship between the pedagogical parameters of the post-method condition, the learning opportunities (CRABBE, 2003; ALLWRIGHT, 2005) and the methodology of projects (BEHRENS, 2006).
\end{abstract}

KEYWORDS: Learning opportunities; Post-method condition; Complexity; Pedagogical parameters of the post-method condition; Methodology of projects. 


\section{Introdução}

Segundo Assmann (1998) vivemos em um mundo em constante evolução de valores, crenças, conceitos e ideias que promove um processo contínuo de mudanças e que requer a revisitação dos paradigmas à nossa volta. Esse movimento repercute na educação requerendo que professores repensem as práticas docentes de caráter simplificador e racionalista do paradigma educacional tradicional que entende o aluno como um espectador e o professor como o detentor e transmissor do saber; prioriza e enfatiza o resultado e não o processo; enxerga as disciplinas da matriz curricular de maneira isolada e fragmentada; minimiza ou ignora a importância das interações e do diálogo; automatiza e desvincula, tendendo a excluir os traços de subjetividade acerca do mundo e das experiências vividas (MORIN, 1990/2008; MORAES, 2006; BEHRENS \& OLIARI, 2007).

Como afirmam Morin (2005/2006), Moraes (2006), Behrens e Oliari (2007) e Mariotti (2007) é necessário buscarmos um novo caminho que nos permita mudar esse panorama e estabelecer tessituras entre o sujeito e o objeto; o professor e o aluno; os acontecimentos da vida, as ações e as interações; a ordem e a desordem, e a estabilidade e o movimento. Além disso, necessitamos enxergar o indivíduo como um ser não apenas racional, mas também provido de sensações, sentimentos, emoções e intuições (MORAES, 2006). Dessa forma, almeja-se substituir uma visão de mundo que racionaliza, dissocia e compartimentaliza por uma perspectiva complexa, ou seja, do que é tecido junto (Morin, 1999/2006a, 2008/2010).

Segundo essa visão, ou seja, da perspectiva da complexidade, a construção de conhecimento é percebida e coproduzida a partir da interação do indivíduo com o objeto, o meio físico e social, por meio de seu diálogo com o mundo (MORIN, 1990/2008), com conceitos e teorias interconectados e em constante transformação (MORAES, 2006). O conhecimento está relacionado às informações e inserido em seu contexto, sendo necessário que aprendamos a articular, organizar, contextualizar e integrar (MORIN, 1999/2006a).

Para D'Ambrósio (2003) o conhecimento ocorre a partir do encontro com o(s) outro(s), sendo gerado individualmente e realizado quando compartilhado. Moraes (2006,) compartilha a visão dos autores e ressalta que a construção de conhecimento é um movimento, um processo de construção e reconstrução mediante processos de assimilação, acomodação, auto-organização e de relações interativas e dialógicas. $\mathrm{O}$ aprender não se relaciona ao memorizar, armazenar e processar dados e informações, mas pesquisar para que possamos desconstruir e depois sermos capazes de reconstruir (TESCAROLO, 2004), pressupondo a participação do indivíduo com seu raciocínio, sua percepção, suas emoções, seus sentimentos, suas sensações e sua intuição.

Morin (1999/2006a, p. 95-96; 2005/2006, p. 74-77) propõe três princípios complementares e interdependentes que nos auxiliam a pensar a complexidade: o 
hologramático,o recursivo e o dialógico O princípio hologramático entende a relação de enriquecimento mútuo entre parte e todo (a parte está no todo bem como o todo na parte) que rompe com ideias lineares, tais como causa/efeito e produto/produtor, pois tudo o que é produzido volta-se sobre o que produz num processo cíclico de construção de conhecimento que é autoconstitutivo, auto-organizador e autoprodutor. O princípio recursivo refere-se à causa agir sobre o efeito e o efeito sobre a causa. $\mathrm{O}$ princípio dialógico permite que assumamos racionalmente a dualidade e a inseparabilidade de noções que parecem contraditórias, tais como ordem ou desordem, razão ou emoção, certo ou errado e que podem ser complementares ou indissociáveis.

Considerando os aspectos mencionados a respeito da perspectiva da complexidade acreditamos que ao pensarmos e implementarmos cursos necessitamos: (1) valorizar e considerar a experiência de todos os indivíduos envolvidos, contando com a razão, as emoções, os sentimentos, as intuições e as sensações; (2) possibilitar a ligação, o diálogo e as interações; (3) aceitar e lidar com a desordem, contradições e a possibilidade do erro; (4) inter-relacionar os eventos de forma que haja uma relação entre as partes, num movimento contínuo, não definível e mensurável, e (5) não enxergar o professor como um indivíduo que detém e transmite o saber, o dono de verdades absolutas (D’ESPOSITO, 2012).

O professor complexo é um intermitente questionador dos problemas e das soluções, capaz de transformar sua forma de pensar, investigar, se relacionar e adotar novas perspectivas (BEHRENS, 2006). Segundo Moraes (2008) cabe a ele uma prática de caráter reflexivo e crítico; identificar necessidades (intuindo e convertendo tudo em subsídios para o ensino e a aprendizagem); estar atento aos processos auto-organizadores dos alunos; ter discernimento e atitude diante dos problemas; ter percepção do momento adequado para mudanças; enfrentar desafios, além de pensar e propor novas estratégias pedagógicas que vão ao encontro dos princípios da complexidade.

Baseado nos aspectos mencionados relacionados à mudança paradigmática na educação e na perspectiva da complexidade, proporemos um diálogo entre a condição pós-método proposta por Kumaravadivelu (2006) examinando a inter-relação entre os parâmetros pedagógicos da condição pós-método, a noção de oportunidades de aprendizagem (CRABBE, 2003 e ALLWRIGHT, 2005) e a metodologia de projetos (BEHRENS, 2006).

\section{Estabelecendo um diálogo entre a condição pós-método e a complexidade}

A proposta de pós-método de Kumaravadivelu (2006) busca uma integração e inter-relação entre teoria e prática, sendo sensível e crítico em relação ao contexto, às condições de aprendizagem e ensino, pois como salienta o autor: 
A condição pós-método é um estado de coisas sustentável que nos obriga a fundamentalmente reestruturar nossa visão do ensino de línguas e da formação de professores. Nos convida a revisar o caráter e o conteúdo do ensino em sala de aula em todas as suas perspectivas pedagógicas e ideológicas. Nos impulsiona a revisitar nossa formação de professores, refigurando a relação reificada entre teoria e prática. (KUMARAVADIVELU, 2006, p. 170, grifo do autor) ${ }^{1}$.

O professor deve observar e estudar atentamente sua prática, sua sala de aula e o contexto como um todo (a escola e seu entorno) para que possa coletar informações e, baseado nelas, posteriormente refletir, interpretar e reformular sua prática com o intuito de possibilitar oportunidades de aprendizagem aos alunos. Assim, as informações coletadas lhe permitirão avaliar os resultados, identificar problemas, encontrar e aplicar possíveis soluções, e novamente observar o que funciona ou não (KUMARAVADIVELU, 2006).

Para tal, Kumaravadivelu (2006) propõe os parâmetros pedagógicos da particularidade, praticalidade e possibilidade. O parâmetro da particularidade enfatiza o valor das experiências vividas e das exigências locais requerendo atenção aos aspectos linguísticos, socioculturais e políticos e uma educação que seja sensível ao contexto. $\mathrm{O}$ parâmetro da praticalidade volta-se à relação entre teoria e prática, com o conhecimento pedagógico do professor emergindo de suas práticas diárias de ensino. Assim, os professores teorizam a partir de sua prática docente e praticam o que teorizam em um ciclo recursivo de construção de conhecimento. Por sua vez, o parâmetro da possibilidade enfatiza que as experiências vividas e trazidas pelos participantes ao ambiente pedagógico são compostas não apenas pelas experiências de sala de aula, mas também por aquelas de âmbito social, econômico e político do mundo que os cerca.

Como salienta o próprio autor, os parâmetros pedagógicos da particularidade, praticalidade e possibilidade "se entrelaçam e interagem em uma relação sinérgica na qual o todo é muito mais do que a soma de suas partes" (KUMARAVADIVELU, 2006, p. 171). Tal afirmação nos remete à complexidade, pois para Morin (1999/2006, p. 89; 2008/2010, p. 190) o complexo não se refere a algo difícil ou complicado, mas ao que é "tecido junto", ou ao que abarca, compreende, abrange, como salienta Tescarolo (2004).

A proposta de pós-método vai ao encontro da complexidade, pois busca a não fragmentação entre teoria e prática, considerando que há uma rede de relações e eventos inter-relacionados. O conhecimento sobre a sala de aula e a prática docente de cada professor é visto, percebido e coproduzido por meio do diálogo do professor com seus

${ }^{1}$ The postmethod condition is a sustainable state of affairs that compels us to fundamentally restructure our view of language teaching and teacher education. It urges us to review the character and content of classroom teaching in all its pedagogical and ideological perspectives. It drives us to streamline our teacher education by refiguring the reified relationship between theory and practice. (Tradução das autoras, pois até o momento a edição não conta com tradução para o português). 
alunos, a sala de aula, o contexto e o mundo, pressupondo e decorrendo da participação e da interação com o objeto, os meios físico e social (MORAES, 2006). A construção e reconstrução de conhecimento são constituídas por verdades mutáveis e imprevisíveis, por meio de enriquecimentos mútuos, nada lineares ou pré-determinados, pois se vivencia o processo de construção de conhecimento exploramos conexões, relações, integrações (MORIN, 2008).

Como ressaltado por Kumaravadivelu (2006) os parâmetros pedagógicos não se excluem, mas se complementam, havendo uma articulação e associação entre eles. Esse aspecto nos remete ao princípio hologramático (MORIN, 1999/2006, 2005/2006) em que não apenas a parte está no todo, como o todo está inscrito na parte, podendo-se enriquecer as partes pelo todo e o todo pelas partes, num mesmo movimento produtor de conhecimentos. O mundo físico da sala de aula passa a ser visto como uma rede de relações, ou seja, todos os eventos estão e são inter-relacionados e a relação entre as partes determina a tessitura de uma teia onde não existem partes isoladas nem uma ordem determinada (BEHRENS e OLIARI, 2007).

Também podemos notar o princípio da recursividade que estabelece que produtos e efeitos são, eles próprios, produtores e causadores daquilo que os produz. Esse princípio se faz presente na prática docente quando, durante o trabalho realizado em sala de aula, somos influenciados e construímos conhecimento a partir do que ocorreu nas aulas anteriores e nossa experiência docente como um todo. Esse conhecimento, por sua vez, retroage sobre nossa prática e nosso trabalho, pois estão imbricados. Assim, retomamos e revemos com um novo olhar nossos atos, avaliando o que ocorreu e seus resultados. Identificamos problemas, buscamos soluções e alternativas baseadas em nossa experiência e o que se dá em nossa sala de aula. Repensamos e modificamos nossa prática docente com o intuito de oferecer oportunidades de aprendizagem aos alunos. Porém, esse movimento recursivo e de construção de conhecimento acerca do que se dá em nossa sala de aula e nossa prática docente não se encerra, havendo sempre uma retomada e reflexão. Dessa forma, nunca retornamos ao mesmo ponto, pois a cada revisitação somos outros docentes, que vivenciaram novas experiências e que, portanto, passam a enxergar sua a prática docente e sala de aula de forma diversa da anterior.

A pedagogia do pós-método atribui significado importante ao aluno em relação à tomada de decisões. Kumaravadivelu (2006) compreende os alunos como indivíduos ativos e autônomos, considerando tanto uma visão estreita quanto uma visão ampla da autonomia. Segundo o autor,

A visão estreita visa desenvolver no aprendiz uma capacidade de aprender a aprender enquanto a visão ampla vai além disso incluindo também a capacidade de aprender a liberar. Auxiliando os alunos a aprenderem que o aprender envolve o desenvolvimento a habilidade "de assumir seu próprio processo de aprendizagem" (Holec, 1981, p.3). Assumir, segundo Holec, significa: (a) ter e ater-se à responsabilidade de determinar os objetivos de aprendizagem, (b) definir conteúdos e 
progressões, (c) selecionar os métodos e técnicas a serem usados, (d) monitorar o procedimento de aquisição, e finalmente, (e) avaliar o que foi adquirido ${ }^{2}$. (p. 176)

O intuito é que os aprendizes tenham consciência sobre o que precisam aprender e possam planejar sua aprendizagem, cientes das estratégias que funcionam para si e que são adequadas naquele determinado momento, responsabilizando-se por sua aprendizagem e se tornando cada vez mais autônomos. Essa autonomia não se restringe ao mundo acadêmico, permitindo ao aprendiz ter competência intelectual, consciência social e atitudes mentais que lhe permitam aproveitar oportunidades e enfrentar desafios dentro e fora da sala de aula.

Em relação ao professor, a pedagogia do pós-método

reconhece o conhecimento prévio do professor bem como o potencial de saber não apenas como ensinar, mas também como atuar autonomamente dentro das limitações administrativas e acadêmicas impostas pelas instituições, currículos, e materiais didáticos. Também promove a habilidade aos professores de saber como desenvolver uma abordagem reflexiva em relação à sua própria prática docente, como analisar e avaliar seus próprios atos, como iniciar uma mudança na sala de aula, e como monitorar os efeitos de tais mudanças (WALLACE, 1991, apud KUMARAVADIVELU, 2006, p. 178)3.

Como salienta Kumaravadivelu (2006) os professores pesquisam sua prática tendo por base o entrelaçamento entre os parâmetros pedagógicos da particularidade, praticalidade e possibilidade. Motivados pelo seu desejo de explorar e melhorar sua

\footnotetext{
${ }^{2}$ The narrow view seeks to develop in the learner a capacity to learn to learn whereas the broad view goes beyond that to include the capacity to learn to liberate as well. Helping learners learn to learn involves developing in them the ability "to take charge of one's own learning" (Holec, 1981, p.3). Taking charge, according to Holec, means to: (a) have and hold the responsibility for determining learning objectives, (b) for defining contents and progressions, (c) for selecting methods and techniques to be used, (d) for monitoring the procedure of acquisition, and finally, (e) for evaluating what has been acquired. (Tradução das autoras, pois até o momento a edição não conta com tradução para o português).

${ }^{3}$ recognizes the teacher's prior knowledge as well as their potential to know not only how to teach but also know how to act autonomously within the academic and administrative constraints imposed by institutions, curricula, and textbooks. It also promotes the ability of teachers to know how to develop a reflective approach to their own teaching, how to analyze and evaluate their own teaching acts, how to initiate change in their classroom, and how to monitor the effects of such changes (Wallace, 1991, apud KUMARAVADIVELU, 2006, p. 178). (Tradução das autoras, pois até o momento a edição não conta com tradução para o português).
} 
própria atuação docente, a pesquisa é iniciada e implementada pelos professores que estão atentos ao que ocorre em sala de aula e abertos a mudanças que permitam que os objetivos sejam atingidos. Ao professor educador, cabe criar "condições para os futuros professores adquirirem a autoridade e autonomia necessárias que os permitirão refletir e delimitar suas experiências pedagógicas, e em certos casos transformar tais experiências" (Kumaravadivelu, 2006, p. 182).

A proposta do pós-método e seu diálogo com a complexidade nos remete à proposta de Allwright (2005) que propõe a substituição do conceito de pontos de ensino das aulas por oportunidades de aprendizagem. Por pontos de ensino o autor refere-se ao conteúdo, ao assunto da aula, o aspecto sobre o qual a aula se desenvolverá e sobre o qual o professor se concentrará. Por sua vez, as oportunidades de aprendizagem, como diz o próprio termo, referem-se às oportunidades que ocorrem dentro e fora de sala de aula $\mathrm{e}$ que permitem ao aluno aprender. Assim, elas são variáveis e um tanto imprevisíveis uma vez que não serão iguais para todos, pois o que pode ser uma oportunidade de aprendizagem para um aluno pode não ser necessariamente para outro, uma vez que aprendemos de formas diversas.

A qualidade da oportunidade de aprendizagem é essencial ao processo de aprendizagem, pois ela é vista como um processo e não um efeito decorrente de uma causa, ou seja, o professor ensina e consequentemente o aluno aprende - visão esta do paradigma simplificador. Existe a necessidade de estarmos cientes de que não existem fórmulas exatas que garantam a aprendizagem, pois os indivíduos são complexos e integrais e o conhecimento visto, percebido e coproduzido por meio do nosso diálogo com o mundo (MORIN, 1990/2008) não só pelo uso da nossa razão, mas também das nossas sensações, nossas emoções, nossos sentimentos e nossas intuições (MORIN 2005/2006; MORAES, 2006; BEHRENS e OLIARI, 2007; MARIOTTI, 2007). A construção do conhecimento ocorre devido a processos transformadores decorrentes das experiências inerentes a cada sujeito e dependentes das ações, interações e transações, ou seja, a relação que estabelecem com o mundo e os demais conhecimentos.

Por sua vez, Crabbe (2003) preocupa-se com nossa compreensão acerca do que sejam oportunidades de aprendizagem, em como torná-las realidade em nossas salas de aula e a conscientização dos docentes e dos aprendizes sobre as mesmas. Segundo o autor cabe ao professor: (1) fornecer e conscientizar os alunos sobre as oportunidades de aprendizagem fora e dentro da sala de aula; (2) contribuir para um ambiente de sala e sócio cultural que seja positivo; (3) fornecer modelos e discussões das diversas abordagens de ensino; (4) incentivar a participação, estruturar objetivos, dar feedback positivo, conscientizar sobre a relação entre processo e produto; (5) ajudar o aluno a estabelecer uma rotina e um comportamento de aprendizagem, e (6) fornecer feedback significativo e encorajar a autoavaliação.

Assim, ao articularmos tais propostas podemos notar a busca por uma interrelação entre a teoria e a prática, e uma prática docente que seja sensível e crítica em relação ao contexto e às condições de ensino e aprendizagem. $\mathrm{O}$ intuito é poder oferecer 
ao aluno oportunidades de aprendizagem que possibilitem a construção de conhecimento por meio da inter-relação e do diálogo entre as diferentes partes que compreendem o sistema do ensino-aprendizagem, do contato com as experiências prévias e vivências de cada um dos indivíduos envolvidos no processo, e a relação que estabelecem com o mundo e os demais conhecimentos. Mas como podemos operacionalizar esse diálogo?

\section{Operacionalizando o diálogo}

Tentando operacionalizar este diálogo entre as oportunidades de aprendizagem, a complexidade e o pós-método, buscamos a Metodologia de Projetos proposta por Behrens (2006) que objetiva promover o desenvolvimento de uma atividade educativa, por meio da investigação de possíveis respostas para um determinado problema que permita a produção de conhecimento com autonomia e espírito crítico. Para a autora a aprendizagem se dá a partir de problematizações que permitem: (1) a busca de caminhos e respostas (requerendo discernimento para se optar e escolher possíveis soluções); (2) o comprometimento de todas as partes envolvidas; (3) visão crítica e ética; (4) a aproximação do aluno a situações que possa vir a enfrentar, e (5) investigações individuais ou coletivas e que permitam aos alunos acessar diversas maneiras de aprender (especialmente de aprender a aprender) sem que haja a memorização de informações.

Baseada na afirmação acima, Behrens (2006) propõe fases para desencadear a Metodologia de Projetos que, como salienta a autora, não são regras que necessitam ser rigorosamente seguidas, nem mesmo fases estanques ou isoladas, uma vez que há um diálogo entre elas. As possibilidades apresentadas são:

1. apresentação e discussão do projeto: permite que haja apreciação e abertura de espaço para sugestões e reconstrução;

2. problematização: desencadeia a discussão e envolve os alunos no tema. Os problemas devem ser retirados da realidade dos alunos e levar em conta suas vivências, interesses e possibilidades de realização de experiências significativas;

3. contextualização: permite ao professor ajudar os alunos a delimitar o objetivo da investigação, alertando-os sobre como buscar informações que poderão contribuir e responder às indagações do problema;

4. aulas dialogadas/teóricas exploratórias: ajudam os alunos a esclarecer caminhos que possam auxiliá-los na aprendizagem significativa;

5. pesquisa individual: permite a busca de informações em diversas fontes;

6. produção individual: possibilita aos alunos manifestarem suas opiniões e apresentarem suas produções a partir das pesquisas que tenham realizado individualmente e compartilhada com os colegas; 
7. discussão coletiva, crítica e reflexiva: permite que os alunos apresentem, defendam e critiquem ideias a partir de suas pesquisas e produções;

8. produção coletiva possibilita acoplar e interconectar as produções individuais;

9. produção final: permite a intervenção na realidade, com professor e alunos discutindo a possibilidade de aplicação das produções individuais e coletivas;

10. avaliação formadora da aprendizagem: permeia todos os momentos do projeto e conta com a participação dos alunos que se manifestam e discutem sobre a avaliação (buscando consenso a respeito dos critérios) e

11. avaliação do projeto: permite aos alunos manifestarem suas opiniões a respeito de suas vivências e experiências ao longo de todo o processo.

Buscando estabelecer uma relação e fazendo uma análise da Metodologia de Projetos (BEHRENS, 2006) e das responsabilidades do professor propostas por Crabbe (2003) notamos ser possível ofertar e conscientizar os alunos sobre as oportunidades de aprendizagem fora e dentro da sala de aula ao longo de todo o desenvolvimento do processo. Tanto os professores quanto os alunos podem conjuntamente principalmente nas fases de problematização, contextualização, avaliação formadora de aprendizagem e de avaliação do projeto construírem esta relação oportunidades de aprendizagem.

Acreditamos que as fases da Metodologia de projetos venham a contribuir para um ambiente de sala e sócio cultural que seja positivo e as fases de aulas dialogadas/teóricas exploratórias, pesquisa e produção individuais, discussão coletiva, crítica e reflexiva, produção coletiva e final; avaliação formadora da aprendizagem e do projeto permitem que modelos sejam fornecidos, analisados e discutidos, além de possibilitar que discussões acerca das diversas abordagens de ensino sejam feitas.

Ao longo de todas as fases o incentivo à participação, a estruturação de objetivos, o fornecimento de feedback que seja positivo e significativo, a conscientização sobre a relação entre processo e produto e o encorajamento de se fazer autoavaliação podem ocorrer. Ademais, as fases de problematização; discussão coletiva, crítica e reflexiva; produção coletiva e de avaliação do projeto permitem potencializar a geração de oportunidades de aprendizagem. Por sua vez, as fases de contextualização, aulas dialogadas/teóricas exploratórias, pesquisa individual, produção individual, discussão coletiva, crítica e reflexiva, produção coletiva, produção final e avaliação formadora da aprendizagem podem auxiliar o aluno a estabelecer uma rotina e um comportamento de aprendizagem.

O quadro a seguir contempla a relação entre as oportunidades de aprendizagem (CRABBE, 2003) e a Metodologia de Projetos (BEHRENS, 2006): 
The ESPecialist: Descrição, Ensino e Aprendizagem, Vol.38 No.2 ago-dez 2017 https://revistas.pucsp.br/esp CThe ESPecialist

\begin{tabular}{|c|c|c|c|c|c|c|}
\hline & \multicolumn{5}{|c|}{$\begin{array}{c}\text { Oportunidades de aprendizagem - responsabilidades } \\
\text { do professor }(\mathrm{CRABBE}, 2003)\end{array}$} \\
\hline & & 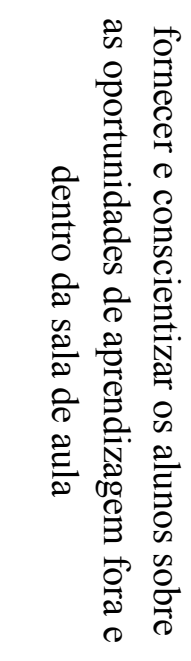 & 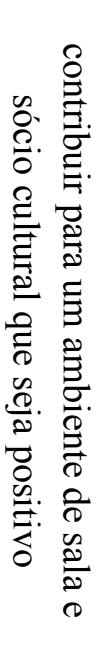 & 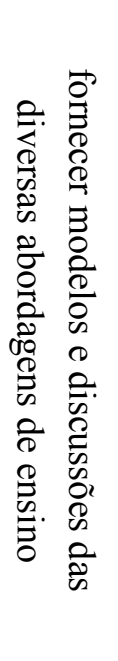 & 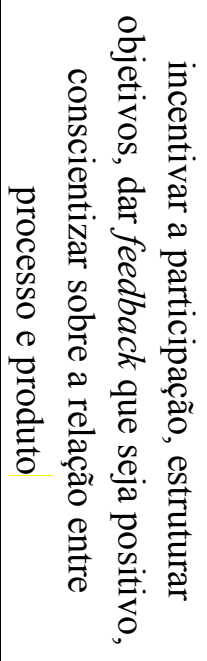 & 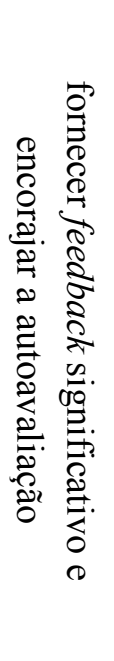 \\
\hline \multirow{11}{*}{ 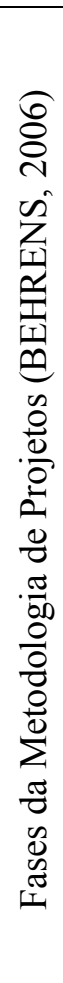 } & Apresentação & $\sqrt{ }$ & $\sqrt{ }$ & & $\sqrt{ }$ & \\
\hline & Problematização & $\sqrt{ }$ & $\sqrt{ }$ & & $\sqrt{ }$ & \\
\hline & Contextualização & $\sqrt{ }$ & $\sqrt{ }$ & & $\sqrt{ }$ & $\sqrt{ }$ \\
\hline & $\begin{array}{c}\text { aulas dialogadas/ } \\
\text { teóricas } \\
\text { exploratórias }\end{array}$ & $\sqrt{ }$ & $\sqrt{ }$ & $\sqrt{ }$ & $\sqrt{ }$ & $\sqrt{ }$ \\
\hline & pesquisa individual & $\sqrt{ }$ & $\sqrt{ }$ & $\sqrt{ }$ & $\sqrt{ }$ & $\sqrt{ }$ \\
\hline & produção individual & $\sqrt{ }$ & $\sqrt{ }$ & $\sqrt{ }$ & $\sqrt{ }$ & $\sqrt{ }$ \\
\hline & $\begin{array}{l}\text { discussão coletiva, } \\
\text { crítica e reflexiva }\end{array}$ & $\sqrt{ }$ & $\sqrt{ }$ & $\sqrt{ }$ & $\sqrt{ }$ & $\sqrt{ }$ \\
\hline & produção coletiva & $\sqrt{ }$ & $\sqrt{ }$ & $\sqrt{ }$ & $\sqrt{ }$ & $\sqrt{ }$ \\
\hline & produção final & & $\sqrt{ }$ & $\sqrt{ }$ & $\sqrt{ }$ & $\sqrt{ }$ \\
\hline & $\begin{array}{c}\text { avaliação } \\
\text { formadora da } \\
\text { aprendizagem }\end{array}$ & $\sqrt{ }$ & $\sqrt{ }$ & $\sqrt{ }$ & $\sqrt{ }$ & $\sqrt{ }$ \\
\hline & $\begin{array}{l}\text { avaliação do } \\
\text { projeto }\end{array}$ & $\sqrt{ }$ & $\sqrt{ }$ & $\sqrt{ }$ & $\sqrt{ }$ & $\sqrt{ }$ \\
\hline
\end{tabular}

Quadro 1 - Relação oportunidades de aprendizagem (CRABBE, 2003) e a Metodologia de Projetos (BEHRENS, 2006)

\section{Considerações Finais}


Neste artigo, após termos resenhado autores que discutem a respeito da mudança paradigmática na educação, apresentamos uma visão em relação ao ensino aprendizagem ao pensarmos e repensarmos acerca das oportunidades de aprendizagem (CRABBE, 2003; ALLWRIGHT, 2005) que oferecemos aos nossos alunos dentro e fora da sala de aula. Sendo esta uma proposta de cunho teórico, apresentamos um diálogo entre a proposta de pós-método (KUMARAVADIVELU, 2006) e a perspectiva da complexidade (MORIN, 1990/2008, 1999/2006a, 1999/2006b, 2005/2006, 2008/2010), examinando a possível inter-relação entre a pedagogia do pós-método, as oportunidades de aprendizagem (CRABBE, 2003; ALLWRIGHT, 2005) e a Metodologia de Projetos (BEHRENS, 2006). Terminamos o artigo plantando uma semente para uma pesquisa futura, ou seja, da investigação a respeito dessa inter-relação que aqui propomos, para assegurarmo-nos que a Metodologia de Projetos seja realmente uma possibilidade de se trabalhar com a condição pós-método. Fica aqui, portanto, um ponto para investigação e trabalhos futuros.

\section{Referências Bibliográficas}

ALLWRIGHT, D. 2005. From teaching points to learning opportunities and beyond. TESOL Quarterly, 39.1: 9-31. Alexandria.

ASSMANN, H. 1998. Metáforas para reencontrar a educação Piracicaba: Unimep.

BEHRENS, M. A. 2006. Paradigma da Complexidade. Metodologia de projetos, contratos didáticos e portfólios. Petrópolis: Editora Vozes.

BEHRENS, M. A.; OLIARI, A. L. T. 2007. A evolução dos paradigmas na educação: do pensamento científico tradicional a complexidade. Diálogo Educacional, 7: 53-66, Curitiba.

CRABBE, D. 2003. The quality of language learning opportunities. TESOL Quarterly, 37.1: 9-34, Alexandria.

D’AMBRÓSIO, U. 2003. Novos paradigmas de atuação e formação de docente. In: T. M. E. PORTO (Org.), 2003, Redes em construção: meios de comunicação e práticas educativas. São Paulo: JM Editora. 1a. ed., pp. 55- 77.

D’ESPOSITO, M. E. W. 2012. Prática Escrita em língua inglesa: um curso online para professores da rede pública estadual, sob a perspectiva da complexidade. Tese de Doutorado, Pontifícia Universidade Católica de São Paulo.

KUMARAVADIVELU, B. 2006. Understanding Language Teaching - from Method to Postmethod. New York: Lawrence Erlbaum Associates.

MARIOTTI, H. 2007. Pensamento complexo: suas implicações à liderança, a aprendizagem e ao desenvolvimento sustentável. São Paulo: Atlas.

MORAES, M. C. 2006. O paradigma Educacional Emergente. Campinas: Papirus.

MORAES, M. C. 2008. Ecologia dos Saberes: Complexidade, transdisciplinaridade e educação. Novos fundamentos para iluminar novas práticas educacionais. São Paulo: Antakarana/WHH- Willis Harman House. 
The ESPecialist: Descrição, Ensino e Aprendizagem, Vol.38 No.2 ago-dez 2017

https://revistas.pucsp.br/esp CThe ESPecialist

ISSN 2318-7115

MORIN, E. 1990/2008. Ciência com consciência. Rio de Janeiro: Bertrand Brasil.

MORIN, E. 1999/2006a. A cabeça bem-feita: repensar a reforma, reformar o pensamento. Rio de Janeiro: Bertrand Brasil.

MORIN, E. 1999/2006b. Os sete saberes necessários à educação do futuro. São Paulo: Cortez Editora.

MORIN, E. 2005/2006. Introdução ao Pensamento Complexo. Porto Alegre: Editora Sulina.

MORIN, E. 2008/2010. Meu caminho. Entrevistas com Djéanne Kareh Tager. Rio de Janeiro: Bertrand Brasil.

TESCAROLO, R. 2004. A escola como sistema complexo: a ação, o poder e o sagrado. São Paulo: Escrituras Editora.

WALLACE, M. J. 1991. Training foreign language teachers: a reflective approach. Cambridge: Cambridge University Press.

Maria Eugenia Witzler D'ESPOSITO is a post-doctoral student in Applied Linguistics and Language Studies at the Pontifical Catholic University of São Paulo (2012). She has a degree in English Language and Literature - Translation and Full Degree in English Language and Literature from the Pontifical Catholic University of São Paulo (1993), Master's Degree in Applied Linguistics and Language Studies from the Pontifical Catholic University of São Paulo (2004) and PhD in Applied Linguistics and Language Studies by the same institution (2012). She has experience in Literature, with emphasis on Modern Foreign Languages, working mainly in the following subjects: teacher training, use of new technologies in education, distance education, written practice, languages for specific purposes, complexity and teaching. She is a member of the research group "Language Teaching and Learning: Approaches, Methodologies and Technologies" (UERJ/CNPq) and member of the Scientific Committee and of the Publications and Promotions / Communications Committee of the International Conference on Information Communication Technologies in Education (ICICTE). Email:eugenia.desposito@facultinglesa.com.br

Maria Antonieta Alba CELANI holds a degree in Anglo Germanic Literature from Pontifical Catholic University of São Paulo (1946), Ph.D. in Anglo Germanic Literature from the Pontifical Catholic University of São Paulo (1960) and Specialization from the University of London and the University of Michigan. She is currently Professor Emeritus of the Pontifical Catholic University of São Paulo. She is part of the editorial staff of the journals the ESPecialist, DELTA, Works in Applied Linguistics and Brazilian Journal of Applied Linguistics. She has experience in the area of Applied Linguistics, with emphasis on teacher education, working mainly in the following subjects: English teaching, public school, teacher development and reflexive formation, relationship theory and practice. She was the founder of the country's first Postgraduate Program in Applied Linguistics in 1970. She coordinated the National Project Teaching Instrumental English in Brazilian Universities (1980-1989) and currently co-ordinates the English Teacher's Continuous Training Program, a partnership between the Associação Cultura Inglesa 
The ESPecialist: Descrição, Ensino e Aprendizagem, Vol.38 No.2 ago-dez 2017 https://revistas.pucsp.br/esp CThe ESPecialist ISSN 2318-7115

São Paulo, PUC-SP, State Secretariat of Education of São Paulo and the Department of Education of the Municipality of São Paulo.E-mail: acelani@pucsp.br 